One should not lose sight of the scale of the problem. We only found two cases in 1758 biopsy specimens where the diagnosis of papillary carcinoma led to resection of a papillary microcarcinoma as well as the benign nodule which was the palpable lesion. Any procedure that increases the chance of finding a clinically insignificant microcarcinoma, such as multiple blind biopsies of non-nodular thyroid tissue or of ultrasound detected lesions of less than $1 \mathrm{~cm}$ in diameter, should be very carefully evaluated before being introduced into routine use. ${ }^{1}$ Multiple blind needle biopsies obviously increase the chance of detecting clinically insignificant lesions-papillary microcarcinomas may occur in as many as $34 \%$ of adult thyroids when they are histologically examined in 2 to $3 \mathrm{~mm}$ steps. If those thyroids were thoroughly examined histologically, it was estimated that about 300 microcarcinomas would have been found. ${ }^{2}$ The prevalence of thyroid cancer will increase still further with an increasing number of sections studied per gland. ${ }^{3}$ Not to mention primary thyroid lesions where fine needle aspiration cytology, serum calcitonin levels or genetic studies helped to yield a diagnosis of an unsuspected medullary microcarcinoma where thyroidectomy should be advocated. ${ }^{4}$

H RUBEN HARACH Department of Histopathology Addenbrooke's Hospital University of Cambridge University of Cambridge, Cambridge CB2 $2 Q Q$

1 Ezzat S, Sarti DA, Cain DR, Braunstein GD. Thyroid incidentalomas. Prevalence by pal-
pation and ultrasonography. Arch Intern Med $1994: 154 \cdot 1838-40$

2 Harach HR, Franssila KO, Wasenius VM. Occult papillary carcinoma of the thyroid. A "normal" papilary carcinoma of the thyroid. A "normal" Cancer 1985;56:531-8.

3 Sampson RJ. Prevalence and significance of occult thyroid cancer. In: DeGroot LJ, ed. $R a$ diation-associated thyroid carcinoma. New York Grune and Stratton, 1977:137-54.

4 Pacini F, Fontanelli M, Fugazzola L, Elisei R, Romei C, DiCoscio G, et al. Routine measurement of serum calcitonin in nodular thyroid diseases allows a preoperative diagnosis of unsuspected sporadic medullary thyroid carcinect 9 Clin Endocrinl $826-9$.

\section{Leukaemia immunophenotyping: effect of antibody source and fluorochrome on antigen detection}

We read with interest the recent pubilication by Howard et al in which the authors highlight discrepant findings of myeloid antigen expression in cases of childhood acute lymphoblastic leukaemia (ALL). They concluded that the detection of antigens CD13 and/or CD33 may be dependent upon both the commercial source of antibody and the type of fluorochrome used. We wish to add support to their conclusions by reporting results from the United Kingdom National External Quality Assurance Scheme (UK NEQAS) for leucocyte immunophenotyping, in addition to data from our own investigations.

Results from UK NEQAS surveys have frequently shown variability in antigen detection attributable, in part, to the use of different commercial monoclonal antibodies. In survey 935, for example (acute bipheno- typic leukaemia), the following mean values for CD13 expression were obtained for each reagent: Becton Dickinson (LeuM7) 15\% $(\mathrm{n}=15)$, Dako $13 \cdot 2 \% \quad(\mathrm{n}=27)$, Coulter $48 \cdot 25 \%(n=8)$, Seralab 3\% $(n=2)$, Ortho $0.5 \%(n=2)$, and Serotec $89 \%(n=1)$. In addition, the scheme has consistently shown statistically significant differences between samples analysed with fluorescein isothiocyanate (FITC) and phycoerythrin (PE) conjugated antibodies for the following antigens: CD3, CD5, CD13, CD14, CD19, and CD33. In survey 923, investigating CD13 detection in a case of acute myeloid leukaemia, eight of 24 laboratories using FITC conjugated antibodies obtained values less than $50 \%$ (overall mean $58 \%$ ), of which three were negative results, as defined as less than $20 \% .^{2}$ In contrast, all 12 laboratories using PE conjugated antibodies obtained results greater than $50 \%$ (mean $77 \%$ ). This variation may be as a result of PE having a higher quantum yield than FITC, thus potentially increasing sensitivity.

In a parallel study to that of Howard and colleagues ${ }^{1}$ we have recently determined the expression of myeloid antigens in $B$ cell chronic lymphocytic leukaemia (B-CLL). As with childhood ALL such "aberrant myeloid" expression has been reported to be of prognostic significance. ${ }^{2}$ To confirm these findings we examined 53 cases of B-CLL (stages 0 to IV), using Becton Dickinson PE conjugated anti-CD13 and anti-CD33 (clones L138 and P67.6, respectively), by whole blood lysis and triple colour staining. In 51 cases fewer than $4 \%$ of B cells expressed either CD13 or CD33 (6\% in two cases) when compared with isotype matched controls. Mean fluorescence staining intensity (MFSI) for both CD13 and CD33 expression did not differ significantly from the negative controls. Previous studies, reporting positive myeloid antigen expression, predominantly used Coulter antiCD13 (MY7) and anti-CD33 (MY9) thus raising the possibility that these discrepant findings may relate to antibody source. To confirm this hypothesis we re-examined 15 of the B-CLL cases with PE conjugated MY7 (CD13) and MY9 (CD33). Of these, nine expressed the CD33 antigen on $\geqslant 10 \%$ of the leukaemic B cells, with five cases being regarded as positive ( $>20 \%$ expression); results in agreement with previous studies. The MFSI values showed a significant increase when compared with controls $(p<0.001)$. No sample expressed CD13 on $>20 \%$ of the leukaemic B cells (one had 12\%) although the values were significantly raised when compared with those obtained using Becton Dickinson antibodies $(p<0.001)$. We feel, therefore, that antibody source and also the fluorochrome used should be taken into account when comparing reports studying "aberrant" myeloid antigen expression.

Data from UK NEQAS, together with our own studies in B-CLL, support the conclusions of Howard and colleagues and raises several important issues. Firstly, which result is right? This question may only be answered if all commercially available reagents are standardised; an important consideration when collecting immunophenotypic data in multicentre trials, particularly if meaningful diagnostic and prognostic information is to be obtained. Secondly the development of newer and more sensitive fluorochromes, coupled with multiparameter technology, will increase the dilemma as to what should be regarded as positive. The simplistic approach using an arbitary cut off point, as suggested in the recent $\mathrm{BSCH}$ guidelines, ${ }^{3}$ will probably not be applicable in the future. Data analysis procedures which currently employ the placement of a cursor at the boundary of the negative population are likely to be inappropriate. More biologically relevant procedures, such as antigen density quantification using calibrated flow cytometers, may yield more meaningful data. ${ }^{4}$ Finally, despite the experience of a number of quality control schemes worldwide, there is no consensus as to the best antibody within a CD group for diagnostic use. Such evaluations would require the production of reference materials which express the antigens of clinical importance. Research in this area is currently under way, ${ }^{5}$ although the technical difficulties must not be underestimated.

$$
\begin{array}{r}
\text { JT REILLY } \\
\text { V GRANGER } \\
\text { PF TEMPERTON } \\
\text { D BARNETT } \\
U K N E Q A S \text { for Leucocyte Immunophenotyping, } \\
\text { Department of Haematology, } \\
\text { Northern General Hospital, } \\
\text { Herries Road, } \\
\text { Sheffield, S5 } 7 A U
\end{array}
$$

1 Howard MR, Thomas L, Reid MM. Variable detection of myeloid antigens in childhood acute lymphoblastic

2 Pinto A, Del Vecchio L, Carbone A, Roncadin M, Volpe R, Serraino D, et al. Expression of myelomonocytic antigens is associated with unfavourable clinicoprognostic factors in Bcell chronic lymphocytic leukaemia. Ann Oncol 1994;2:107-13.

3 British Committee for Standards in Haematology. Immunophenotyping in acute leukaemias. $\mathscr{f}$ Clin Pathol 1994;47:777-81.

4 Lavabre-Bertrand T, Janossy G, Exbrayat C, Bourguard P, Duperray C, Navarro M. Leukaemia associated changes identified by quantitative flow cytometry. II. CD5 overexpression and monitoring in B-CLL. Leukemia 1994;8:1557-63.

5 Barnett D, Granger V, Mayr P, Reilly JT. Stabilised whole blood preparation-An innovative product for external quality assurance in lymphocyte subset analysis. $\mathrm{Br} \mathcal{F}$ Haematol 1994;86 (Suppl 1):91.

Recurrent thombotic occlusions of arteries and veins caused by intravascular metastatic adenocarcinoma

I refer to the case reported by Levi et al ${ }^{1}$ of a young woman with recurrent vascular occlusions found at necropsy to be caused by microscopic metastatic adenocarcinoma. They rightly suspected the presence of malignant disease during life, but despite wideranging invasive, radiological and laboratory investigations were unable to confirm their clinical suspicion. In their last sentence, the authors speculate that cytological examination of the blood, using specific markers for malignant cells, might have detected the adenocarcinoma cells; this may have been so, but I wonder if they performed bone marrow trephine biopsy as it is not mentioned amongst the many investigations listed in their paper. Similarly, no mention was made of bone marrow studies carried out on postmortem tissues.

It is thought that one large trephine biopsy or bilateral biopsies can provide a detection rate of metastatic disease somewhere in the region of $60 \%$. Certainly, it is a worthwhile investigation in the type of case reported by Levi et al and if performed might have resulted 